\title{
Controversies in the management of endometrial cancer 2012
}

\author{
Fuat Demirkiran \\ Division of Gynacologic Oncology, Cerrahpasa Medical Faculty, Istanbul University, Istanbul, Turkey \\ Email: fuatdemirkiran@hotmail.com
}

Received 4 August 2012; revised 2 September 2012; accepted 11 September 2012

\begin{abstract}
Endometrial cancer (EC) is the most frequent cancer of the female genital tract, especially in developed countries and the seventh most common cause of death from cancer in women in Western Europe. Major prognostic factors related to endometrial cancer are stage, grade, and depth of myometrial invasion and the presences of lymho-vascular space invasion. Standard treatment of EC consist of surgical and then adjuvant therapy on the basis of pathological prognostic factors. Standard surgical approach for stage I-II endomerial cancer is total hysterectomy and bilateral salpingo-oophorectomy with or without staging. Without question a comprehensive surgical staging procedure similar to that for ovarian carcinoma should be performed for non-endometrioid EC (uterine papiller serous carcinoma and clear cell adenocarcinoma) due to the aggressive clinical behavior. In contrast, there are some controversial issues in the surgical management-staging of EC and the role of ommentectomy and lymphadenectomy are the issues of current debate.
\end{abstract}

Keywords: Endometrium Cancer; Management

\section{INTRODUCTION}

Endometrial cancer (EC) is the most frequent cancer of the female genital tract, especially in developed countries and the seventh most common cause of death from cancer in women in Western Europe. This malignancy is advanced age disease and more than $90 \%$ of cases occur in women older than 50 years of age. Nearly $5 \%$ of endometrial cancers are associated with a genetic alteration, nonpolyposis colorectal cancer syndrome (Lynch II syndrome) which is associated with germline mutations to DNA mismatch repair genes [1]. Those with this syndrome have a life time risk of developing endometrial cancers of $30 \%-60 \%$.

There are two types of EC on the basis of clinical, pathological and molecular features. The most common type is Type I EC (endometrioid adenocancer) which consists of nearly $85 \%$ - $90 \%$ of EC. Second type is nonendometrioid endometrial cancer (Type II) which account for about $10 \%$ of EC. The most common nonendometrioid histology is papillary serous $(10 \%)$, followed by clear cell ( $2 \%$ to $4 \%$ ), mucinous ( $0.6 \%$ to $5 \%$ ), and squamous cell $(0.1 \%$ to $0.5 \%)$ [2]. Features of the type I carcinomas include increased exposure to estrogen (nulliparity, early menarche, chronic anovulation, and unopposed exogenous estrogen) and obesity. The most of type I EC are low grade and good prognostic tumor. In contrast with, type II cancer often arises in those who are multiparous, and not obese women and the prognosis of these tumors is worse.

Major prognostic factors related to endometrial cancer are stage, grade, and depth of myometrial(MI) invasion and the presences of lymho-vascular space invasion (LVSI). Definitive staging according to the FIGO system is based on surgical and pathology findings. In 2009, the revised FIGO staging system was published [3]. At this new reversing system, FIGO 1988 stage IA and IB have been grouped together in FIGO 2009 as stage IA, and FIGO 1988 stage IC is IB in FIGO 2009. Also FIGO 1988 stage IIIC has been divided to two groups, IIIC1 the tumor disseminated pelvic lymph nodes and IIIC2 the tumor disseminated to para-aortic lymph nodes.

EC has been classified three risk group, as low-risk, intermediate-risk, and high-risk for lymph node metastases and/or early disease spread to the abdominal cavity and to distant sites.

Low Risk Group: FIGO 2009 stage IA , grade 1 or 2, of endometrioid type histology.

Intermediate Risk Group: FIGO 2009 stage IA of grade3, stage IB of grade 1or 2 of enodometrioid type histology.

High Risk Group: FIGO 2009 stage IB of grade 3 or of non-endometrioid histology; or stage II or III EC.

\section{DIAGNOSIS}

Abnormal uterine bleeding is the most common symptom of EC and it is usually presented at the beginning of 
disease. Diagnostic work-up should be started with pelvic ultrasound and then followed up by endometrial biopsy in premenopausal and postmenopausal period. Postmenopausal bleeding is usually evaluated by endometrial biopsy because of malignancy rate is more than $10 \%$ [4]. In premenopausal period, malignancy rate in women presenting abnormal uterine bleeding is less than $2 \%$, so in that group of patients, age and ultrasonographic findings of endometrium affect the decision of biopsy [5]. A meta-analysis reported that the Pipelle was the best endometrial sampling device, with detection rates for endometrial cancer in postmenopausal and premenopausal women of $99.6 \%$ and $91 \%$, respectively [6]. A recent study concluded that diagnostic accuracy of D\&C and piplle biopsy are nearly equal, and the first step in diagnostic pathway should be evaluation endometrial morphology and the measurement of endometrial thickness [7]. Also, saline infusion sonography should be used to detect focal lesions which are most common reason of high false negative rate of $\mathrm{D} \& \mathrm{C}$ and Pipelle biopsy between focal and diffuse pathology $[7,8]$.

\section{TREATMENT}

Standard treatment of EC consist of surgical and then adjuvant therapy on the basis of pathological prognostic factors. Standard surgical approach for stage I-II endomerial cancer is total hysterectomy and bilateral salpingo-oophorectomy with or without staging (Table 1).

Without question a comprehensive surgical staging procedure similar to that for ovarian carcinoma should be performed for non-endometrioid EC (uterine papiller serous carcinoma and clear cell adenocarcinoma) due to the aggressive clinical behavior [9]. In contrast, there are some controversial issues in the surgical managementstaging of endometrioid EC and the role of ommentectomy and lymphadenectomy are an issues of current debate.

\section{STAGING SURGERY IN ENDOMETRIOID EC}

Many clinical data has shown us that surgical staging should be main part of the surgical procedure of EC since the high rate of intra-peritoneal and retroperitoneal tumor spreading has been found with surgical staging in

Table 1. Standart surgery for endometrial cancer.

Hysterectomy
Bilateral salpingo-oophorectomy
Staging surgery
Intraperitoneal staging (Ommentectomy, peritoneal biopsy)?
Retroperitoneal staging (Pelvic and/or paraaortic
lymphadenectomy)?

patients with early stage endometrial cancer. At one of the oldest GOG studies published 1984, it was suggested that tumor is beyond to uterus in the $46 \%$ of patients having clinical stage I EC [10]. In GOG 33, authors reported that at the time of surgery, women with apparent stage I tumors spread outside of the uterus in approximately $20 \%$ of cases [11]. In another study published 1990, up-staged ratio of clinical stage I EC was found as $25 \%$ following to surgical staging [12]. Saygllı et al. reported extra-uterine involvement in $22 \%$ of patients (22/97) with clinically stage I disease, and they concluded that positive peritoneal cytology, pelvic lymph node involvement, adnexial involvement, and omental metastases were the most common types of extra-uterine involvement [13]. A prospective study published by Fujiwara at 2008 was performed on 134 patients with clinical stage I EC and all patients underwent surgical staging [14]. At this study, the frequency of omental, adnexial and nodal metastases were $3 \%, 7.5 \%$ and $10 \%$, respectively. These results show us the importance of staging procedure in the surgical management of EC.

\section{PERITONEAL STAGING}

This procedure is the first step of the staging surgery, and omentectomy, biopsies from suspicious intrapertitoneal regions and appendectomy are main parts of this surgical approach. Actually, there is a lack of consensus on the extent of surgical peritoneal staging of endometrioid EC. There have been very few reports addressing the therapeutic value of omentectomy for the treatment of EC, particularly for endometrioid adenocarcinoma. In a recent study, Fujiwara et al. found omental involvement in the 3\% of clinical stage I endometrioid EC [14]. With this result, they concluded that omental metastases rate for clinical stage I endometrioid adenocarcinoma was lower than the positive rates for extrauterine spread to other sites; thus, the routine application of omentectomy as a part of a staging laparotomy may not be efficacious. The reported frequency of omental metastases in clinical stage I endometrial cancer ranged from $6.0 \%$ to $8.3 \%$ $[13,15,16]$. However, nonendometrioid adenocarcinomas were included in these reports. When nonendometrioid adenocarcinomas, such as serous adenocarcinoma, were excluded, the frequency of omental metastases decreased to $3.1 \%$ and $5.3 \%[13,15]$. Chen reported that metastases were confirmed by histologic examination in 3 of 202 cases $(1.5 \%)$ diagnosed to be free of metastases by intraoperative inspection and palpation [17] . In another study published 2003, Gehrig et al. reported that most cases of omental metastases could be detected by inspection because the sensitivity was 0.89 and the specificity was 1.00 for the intraoperative diagnosis of endometrial serous adenocarcinoma via surgical inspection [18]. Also most of omental metastases are associated 
with high grade, deep MI and lymphatic involvement.

Many clinico-pathologic studies clarified that the frequency of omental metastases was lower than that of lymph node metastases and positive peritoneal cytology in clinical stage I endometrioid adenocarcinoma. Thus, performing omentectomy on all patients as a part of routine laparotomy is discussable. In patient having high grade tumor, at least infra-gastric omentectomy or large omental biopsy should be a part of surgical staging.

\section{LENFADENECTOMY (LA) (RETROPERITONEAL STAGING)}

Lymph node metastasis is the most important prognostic factor in early stage EC, but pelvic with or without para-aortic lymph node dissection has been a subject of continuous debate in the surgical management of EC since many years. There are many controversial issues related to LA. These are the balance between cost and benefit, intraoperative complications and postoperative sequelae of this procedure, such as lymphocele, lymphorrhea and lymphedema. The evidence supporting LA in the surgical management of EC originally came from GOG study published at 1987 [11]. This study prospectively evaluated 621 patients with clinical stage I endometrial cancer. Authors reported that incidence of pelvic and para-aortic lymph node metastasis changed from $0 \%$ to $61 \%$ and $0 \%$ to $30 \%$ according to risk factors such as grade, MI and intraperitoneal involvement. In this study, for low risk group the risk of nodal spread was $4 \%$ for the pelvic nodes and 2\% for para-aortic nodes. For the high risk patients (Grade 3 tumors or greater than 50\% uterine wall involvement) the rate was $16 \%$ for pelvic nodes and $10 \%$ for para-aortic nodes. In another study published at 1991, of patients with clinical stage I disease, about $10 \%$ had pelvic and $6 \%$ had para-aortic lymph node metastases [19]. The rate of positivity of both pelvic and para-aortic lymph nodes ranges from 3\% to $51 \%[11,20]$. Some studies founded that no patient with endometrial grade 1 or 2 tumors and $<50 \%$ myometrial invasion had lymph node metastasis when the tumor size was equal or less than 2 centimeters [21,22]. In a study published at 2009, Abu Rustum et al. reported a rate of $1 \%$ to $1.6 \%$ of isolated para-aortic lymph node involvement in the setting of negative pelvic lymph nodes and found this to be consistent for both low- and high-grade lesions [23]. In contrast, in a study from Mayo Clinic, authors prospectively evaluated 281 patients undergoing lymphadenectomy for endometrial cancer staging and found $22 \%$ of patients with high-risk disease had lymph node metastases [20]. Of these, 51\% had both pelvic and para-aortic node metastases, 33\% had positive pelvic lymph nodes only, and $16 \%$ had isolated para-aortic node metastases. In those with para-aortic lymph node involvement, $77 \%$ had metastases above the inferior mesen- teric artery. Conversely, they found that patients with low grade disease (i.e., grade 1 and 2 endometrioid lesions with myometrial invasion $<50 \%$ and tumor size $\leq 2 \mathrm{~cm}$ ) had no lymph node metastases. However, there is no any study supporting these high rates of para-aortic metastases in the literature.

\section{PROGNOSTIC ROLE OF LYMPHADENECTOMY}

There are close relation between nodal metastases and recurrence rate and survival in patients with EC and obviously, strongest predictor of tumor recurrence usually is lymph node status. In an old study, Morrow et al. using the GOG 33 database reported that the five-year recurrence-free survival was $90 \%$ in patients without lymph node metastases, $75 \%$ in patients with pelvic lymph node metastases, and 38\% in patients with positive para-aortic lymph nodes [19]. In a study published 2007, Fajimato et al. found that the five-year survival rate for patients with pelvic without para-aortic node metastases was $84 \%$ compared with $44 \%$ for patients with para-aortic with/or without pelvic node metastases [24]. Mariani et al. noted that just the presence or absence of paraaortic lymph node metastases was of paramount importance [25]. Of para-aortic node-positive patients, 58\% developed progressive or recurrent cancer, and $90 \%$ of patients with a paraaortic recurrence died of disease. In another study, from Chicago USA, it was reported that 5-year survival was $70 \%$ for patients with pelvic node metastases only and $49 \%$ for those with para-aortic node metastases [26].

Although these studies showing clear association between nodal status and end-point results, the therapeutic benefit of pelvic and para-aortic LA or lymph node sampling has been widely debated. Without question, the main purpose of LA is the detection of lymphatic spread that would allow appropriate adjuvant therapy and to increase cure rate of patients.

Some observational studies have compared outcomes in early-stage endometrial cancer patients with and without systematic LA. Retrospective, some single institution studies advocate LA for all grades of tumor [2729]. For example, in 1995, Kilgore et al. published retrospective results of 649 patients with EC. Of these patients, 212 underwent multiple-side lymph node sampling and 205 patients had limited pelvic node sampling (less than four pelvic sites), while 208 patients were not sampled. They concluded that overall survival was significantly better in patients with multiple-site lymph node sampling [27]. A large series utilizing a national database supported lymph node dissection for grade 3 tumors only, with no benefit seen in grade 1 or 2 tumors [30]. From the SEER database, Chan et al. found that in the intermediate/high-risk patients (Stage IB, grade 3; 
Stage IC and II-IV, all grades), lymph node dissection was associated with improved 5-year disease-specific survival and there was no survival benefit seen in patients with low-risk disease [31]. In another study published by Chan et al. they noted no survival benefit for women with stage I, grade 1 disease in LA group [32]. Similarly, Neubauer et al. found no survival benefit to lymph node dissection in patients with preoperative grade 1 endometrial cancer [33]. In a 2007 analysis of the large SEER database from the United States, 42,814 patients with endometrial adenocarcinoma were analyzed by multivariate Cox regression analysis and lymphadenectomy was identified as an independent prognostic factor for overall survival [34]. In a randomized study (SEPAL) involving 2 institutions from Japon published at 2010, 671 patients were randomized to pelvic lymph node dissection with pelvic-paraaortic lymph node dissection [35]. SEPAL study have shown that paraaortic lymphadenectomy has survival benefit to for patients at intermediate or high risk of recurrence, and that pelvic lymphadenectomy alone might be an insufficient surgical procedure for endometrial cancer in patients at risk of lymph node metastasis. In a recent study, Lowery et al., using the SEER database, reported that in women older than 80 , systematic lymphadenectomy was associated with improved survival for high grade, but similar survival for low grade endometrial cancer, consistent with what is seen with younger women and they conclude that the surgeon should carefully weigh the surgical risks and benefits in this patient population, which may be at higher risk for morbidity [36]. Also in this trial, authors found a trend toward improved survival only in the grade 3 cohort in all age groups examined.

Many factors, such as removed lymph node counts, affect the benefit and therapeutic role of LA in the surgical management of EC. Although individual lymph node counts vary considerably between patients and pathologists, data have been published that show a high correlation between the average number of nodes reported in a study with the rate of positive lymph nodes in patients with apparent stage I endometrial cancer [37]. Two studies suggested that one would need an average of approximately 20 lymph nodes to detect most nodal spread $[38,39]$. Other some studies reported that benefit for LA depended on the number of lymph nodes removed at the time of surgery [31,40]. However, there are no randomized trials supporting the benefit of LA in early-stage endometrial cancer. Unfortunately, there is no any standard method to count lymph nodes and essential removed counts for optimal benefit. At the same time, the extent of LA, its therapeutic role, and the definition of an "adequate" LA based on pathologic parameters, such as lymph node counts and anatomic templates, continues to be debated by the gynecologic oncology and radiation oncology physicians who treat women with endometrial carcinoma.

Recently, two large randomized clinical studies addressed the utility of lymphatic staging in the surgical therapy of EC. The first one published by Panici et al. 2005 and authors concluded no improvement in survival for women after pelvic lymphadenectomy, although there was a marginal decrease in the use of postoperative adjuvant radiation therapy after a staging procedure [41]. The ASTEC study was a randomized controlled trial involving 85 institutions in 4 countries [38]. In this study 1408 patients were randomized to abdominal hysterectomy, bilateral salpingo-oophorectomy with or without pelvic lymph node dissection and then subsequently to the use of postoperative radiation. Similarly, the results of the ASTEC study suggested that there was no benefit from either pelvic LA or postoperative pelvic radiation [38]. There some criticisms about power of these two trials. For example, in fist trial from Italy, there was no systematic para-aortic LA, $42 \%$ of patients were low risk group and decision of adjuvant radiotherapy was not homogenous. However, the rates of positive nodes were lower in the ASTEC trial than those reported in other studies [11,39]. In ASTEC study, the rate of patients with low risk was $44 \%$ and the LA versus no dissection arms were unbalanced in terms of high-risk criteria There were 3\% more high-risk histologies, 3\% more high-grade lesions, 3\% more lymphovascular space invasion, and $10 \%$ more deep myometrial invasions in the LA arm despite randomization. This difference may appear small, but could have affected the power of the study to detect differences in survival [42,43]. In both studies, the number of cases with positive lymph nodes was not enough for comparing the results. The ASTEC study had only 27 patients in the LA group that had positive lymph nodes despite enrolling over 700 women in each arm. The Italian trial did have a better detection rate, but only 35 patients in this trial had positive nodes in the LA arm. Also, para-aortic nodes were not sampled in the majority of patients in both of this trial and LA in both studies was not systematic, and did not remove important regional lymph nodes for EC.

Despite FIGO surgical staging, several observational studies which suggested the benefit therapeutic effect of LA, and two large randomized trials which concluded no improvement in survival for women with early stage EC after pelvic lymphadenectomy, comprehensive surgical staging for endometrial cancer is still controversial and there is no clear consensus. Except a few studies, most of them actually are non randomized trials, not having standard staging technique and also for most of them it is not clear limitations and regions of LA. So it is not easy to come to conclusion the therapeutic role of LA in the management of EC. When we look at the clinical 
practice, we see that there are proponents of no LA, pelvic LA only, and complete pelvic and para-aortic lymph node dissection. The proponents of pelvic with or without para-aortic staging and intraperitoneal staging argue that a significant proportion of patients will be upstaged or upgraded on final pathologic report, and that knowing the status of the lymph nodes and intraperitoneal cavity will lead to a more judicious use of adjuvant therapy. If it is thought all type of endometrial cancer, Grade 3 endometrioid cancer, papillary serous cancer, clear cell cancer, undifferentiated, and squamous histologies have a poorer prognosis and may merit comprehensive surgical staging, and the advantages of this advanced surgery outweigh the disadvantages in these high-risk histologies. In our own practice we individually tailor decisions about comprehensive surgical staging based on preoperative grade and MI, intraoperative gross findings, and each patient's co-morbid conditions. We do peritoneal staging and systemic pelvic LA for all EC and we added systemic para-aortic LA for grade 3, depth MI and non-endometrioid tumors.

\section{LYMPHADENECTOMY BASED ON INTRA-OPERATIVE EVALUATIONS}

In some clinics, authors suggest that LA should be done according to intraoperative frozen-section (FS) findings [20]. But, there are some controversial on FS results for performing and tailoring of LA. For example, at 2004 Frumovitz et al showed that FS analysis of tumor grade and depth of myometrial invasion are not always concordant with that of permanent sections [44]. Also two other studies reported a $5 \%-7 \%$ risk of suboptimal surgical treatment of endometrial cancer patients when FS analysis is considered as the basis of surgical management $[45,46]$. In a study published 2009 by Pristauz et al., authors reported that intraoperative frozen section histology missed nearly two of three endometrial cancer patients with positive nodes and results do not support tailoring the extent of lymphadenectomy according to the results of frozen section examination [47]. Briefly, most of literature data display that FS is poor indicator of pathological prognostic factors compared with that of final permanent section report. LA based on FS findings may only work, however, in select centers that have access to a dedicated gynecologic pathologist who can provide accurate intraoperative assessment thereby decreasing the chance of significant upstaging.

Nowadays, lymphadenectomy based on sentinel node evaluation is also highly discussable subject and it is not a routine procedure. There are many studies addressing sentinel lymph node detection in the surgical management of EC $[48,49]$. Regarding to these small number studies, Sentinel lymph node detection cannot yet be considered equal to a complete lymphadenectomy in endometrial cancer. Larger studies are needed to determine the best method of dye injection, the optimal patient this procedure will benefit, and the efficacy of sentinel lymph node detection in endometrial cancer.

\section{SURGICAL APPROACH: LAPARATOMY-LAPAROSCOPY- ROBOTIC SURGERY}

The traditional surgical technique for EC is laparotomy with midline abdominal incision. Following the first laparoscopic pelvic/para-aortic lymph node dissection for gynecologic cancer, reported by Querleu et al., there has been increasing utilization of the laparoscopic approach for endometrial cancer staging [50,51]. The first randomized GOG study (LAP2) published at 2009 by Walker et al. [39]. The study enrolled 1696 patients to the laparoscopy group and 920 to the laparotomy group. In this study, there was no significant difference in node positivity rate (9\%), positive cytology rate, or detection of advanced stage disease (17\%) and laparoscopy group was found to have an improved safety profile with fewer postoperative complications, less antibiotic use and shorter hospital stay. With these result authors concluded that laparoscopy was feasible and beneficial for the surgical management of endometrial cancer. In a randomized study, safety of laparoscopy versus laparotomy in early stage EC surgery was evaluated and authors concluded that there is no evidence of a benefit for laparoscopic hysterectomy over total abdominal hysterectomy in terms of major complication [52]. Bijen et al. reported that in obese patients with a BMI > $35 \mathrm{~kg} / \mathrm{m}^{2}$ total laparoscopic hysterectomy is not cost effective because of the high conversion rate [53]. In another study, authors showed that Laparoscopy was associated with decreased rates of surgical site infections but had an increased risk of peripheral sensory nerve deficits and lymphedema when compared with laparotomy [54]. Endpoint results of GOG LAP2 study published at 2011 [55]. In this study, it is found that the actual recurrence rates were substantially lower than anticipated, resulting in an estimated 3-year recurrence rate of $11.4 \%$ with laparoscopy and $10.2 \%$ with laparotomy and the estimated 5 -year overall survival was almost identical in both arms at $89.8 \%$. A latest study published at 2012 suggested that laparoscopic endometrial cancer staging is associated with a lower occurrence of both asymptomatic and symptomatic lymphoceles compared to open surgery [56].

The first report of robotic-assisted laparoscopic hysterectomy was published in 2002 [57]. Since that time the use of robotic-assisted laparoscopy for endometrial cancer staging has advanced rapidly, particularly in some European country and United States. The largest published series of robotic-assisted laparoscopic endometrial cancer staging was reported in 2011 by Paley et al. The 
series compared 377 robotic surgeries for endometrial cancer to 131 laparotomies performed at the same institution [58]. The results of this study are consistent with the findings of the LAP2 study. In another study consist of 97 patients, authors found that operating time, and pelvic-paraaortic lymph node counts were not statistically different [59]. Review of the literature has shown an improvement in perioperative outcomes by the robotic surgical approach compared to the traditional open approach, with the exception of operating time [60,61].

Although prospective data on long-term oncologic outcomes of patients undergoing minimally invasive surgery for treatment of endometrial cancer have yet to mature, most of gynecologic oncologists believe that laparoscopic and open surgery are surgically equivalent. Multiple prospective and retrospective studies have shown that laparoscopic surgery is equal to open surgery in terms of adequacy of surgical resection and lymph node counts and have shown that laparoscopic surgery is associated with decreased risk of intraoperative and postoperative complications. However, with the World wide use of endoscopic surgery in the surgical management of EC, its prognostic role will be become clear in the future, so the actual role of this surgery should be investigated further. Until that time laparoscopic or robotic surgery should be done by experienced teams for patients, especially with intermediate and high risk endometrial cancer.

\section{REFERENCES}

[1] McMeekin, D.S., Alektiar, K.M. and Sabbatini, P.J. (2009) Corpus: Epithelial tumors. In Barakat, R.R., Markman, M., Randall, M.E., Eds., Principles and Practice of Gynecologic Oncology, Lipponcott, Philadelphia, 683-732.

[2] Mendivil, A., Schuler, K.M. and Gehrig, P.A. (2009) Non-endometrioid adenocarcinoma of the uterine corpus: A review of selected histological subtypes. Cancer Control, 16, 46-52.

[3] Pecorelli, S. (2009) Revised FIGO staging for carcinoma of the vulva, cervix, and endometrium. International Journal of Gynecology and Obstetrics, 105, 103-104. doi:10.1016/j.ijgo.2009.02.012

[4] Lidor, A., Ismajovich, B., Confino, E. and David, M.P. (1986) Histopathological findings in 226 women with post-menopausal uterine bleeding. Acta Obstetricia et Gynecologica Scandinavica, 65, 41-43. doi:10.3109/00016348609158227

[5] Soguktas, S., Cogendez, E., Kayatas, S.E., Asoglu, M.R, Selcuk, S. and Ertekin, A. (2012) Comparison of saline infusion sonohysterography and hysteroscopy in diagnosis of premenopausal women with abnormal uterine bleeding. European Journal of Obstetrics, Gynecology and Reproductive Biology, 161, 66-70. doi:10.1016/j.ejogrb.2011.11.022

[6] Dijkhuizen, F.P., Mol, B.W., Brölmann, H.A. and Heintz
A. (2000) The accuracy of endometrial sampling in the diagnosis ofpatients with endometrial carcinoma and hyperplasia: A meta-analysis. Cancer, 89, 1765-1772. doi:10.1002/1097-0142(20001015)89:8<1765::AID-CNC $\underline{\mathrm{R} 17>3.3 . \mathrm{CO} ; 2-6}$

[7] Demirkiran, F., Yavuz, E., Erenel, H., Bese, T., Arvas, M. and Sanioglu, C. (2012) Which is the best technique for endometrial sampling? Aspiration (pipelle) versus dilatetion and curettage (D\&C). Archives of Gynecology and Obstetrics, in press. doi:10.1007/s00404-012-2438-8

[8] Cepni, I., Ocal, P., Erkan, S., Saricali, F.S., Akbas, H., Demirkiran, F., Idil, M. and Bese, T. (2005) Comparison of transvaginal sonography, saline infusion sonography and hysteroscopy in the evaluation of uterine cavity pathologies. The Australian \& New Zealand Journal of $\mathrm{Ob}$ stetrics \& Gynecology, 45, 30-35. doi:10.1111/j.1479-828X.2005.00336.X

[9] Chan, J.K., Loizzi, V. and Youssef, M. (2003) Significance of comprehensive surgical staging in noninvasive papillary serous carcinoma of the endometrium. Gynecologic Oncology, 90, 181-188. doi:10.1016/S0090-8258(03)00195-1

[10] Boronow, R.C., Morrow, C.P., Creasman, W.T, Disaia, P.J, Silverberg, S.G., Miller, A. and Blessing, J.A. (1984). Surgical staging in endometrial cancer: Clinical-pathologic findings of a prospective study. Obstetrics and $G y$ necology, 63, 825-832.

[11] Creasman, W.T., Morrow, C.P, Bundy, B.N., Homesley, H.D., Graham, J.E. and Heller, P.B. (1987) Surgical pathologic spread patterns of endometrial cancer. A Gynecologic Oncology Group Study. Cancer, 60, 20352041.

doi:10.1002/1097-0142(19901015)60:8+<2035::AID-CN CR2820601515>3.0.CO;2-8

[12] Ayhan, A., Yarali, H., Urman, B., Yüce, K., Günalp, S. and Havlioglu, S. (1990) Comparison of clinical and surgical-pathologic staging in patients with endometrial carcinoma. Journal of Surgical Oncology, 43, 33-35. doi:10.1002/jso.2930430109

[13] Saygili, U., Kavaz, S., Altunyurt, S., Uslu, T., Koyuncuoglu, M. and Erten, O. (2001) Omentectomy, peritoneal biopsy and appendectomy in patients with clinical stage I endometrial carcinoma. International Journal of Gynecological Cancer, 11, 471-474. doi:10.1046/j.1525-1438.2001.01065.X

[14] Fujiwara, H., Saga, Y., Takahashi, K., Ohwada, M., Enomoto, A., Konno, R., Tanaka, A. and Suzuki, M. (2008) Omental metastases in clinical stage I endometrioid adenocarcinoma. International Journal Gynecological Cancer, 18, 165-167. doi:10.1111/j.1525-1438.2007.00961.x

[15] Chen, S.S. and Spiegel, G. (1991) Stage I endometrial carcinoma. Role of omental biopsy and omentectomy. Journal of Reproductive Medicine, 36, 627-629.

[16] Nieto, J.J., Gornall, R., Toms, E., Clarkson, S., Hogston, P. and Woolas, R.P. (2002) Influence of omental biopsy on adjuvant treatment field in clinical stage I endometrial carcinoma. BJOG, 109, 576-578. doi:10.1111/j.1471-0528.2002.01272.x

[17] Chen, S.S. (1995) Extrauterine spread in endometrial 
carcinoma clinically confined to the uterus. Gynecologic Oncology, 21, 23-31. doi:10.1016/0090-8258(85)90228-8

[18] Gehrig, P.A., Van Le, L. and Fowler Jr., W.C. (2003) The role of omentectomy during the surgical staging of uterine serous carcinoma. International Journal of Gynecological Cancer, 13, 212-215.

doi:10.1046/j.1525-1438.2003.13038.x

[19] Morrow, C.P., Bundy, B.N., Kurman, R.J., Creasman, W.T., Heller, P., Homesley, H.D. and Graham, J.E. (1991) Relationship between surgical-pathological risk factors and outcome in clinical stage I and II carcinoma of the endometrium: A Gynecologic Oncology Group study. Gynecologic Oncology, 40, 55-65. doi:10.1016/0090-8258(91)90086-K

[20] Mariani, A., Dowdy, S.C., Cliby, W.A., Gostout, B.S., Jones, M.B., Wilson, T.O. and Podratz K.C. (2008) Prospective assessment of lymphatic dissemination in endometrial cancer: A paradigm shift in surgical staging. Gynecologic Oncology, 109, 11-18. doi:10.1016/j.ygyno.2008.01.023

[21] Schink, J.C., Lurain, J.R., Wallemark, C.B. and Chmiel, J.S. (1987) Tumor size in endometrial cancer: A prognostic factor forlymph nodemetastasis. Obstetrics and Gynecology, 70, 216-219.

[22] Mariani, A., Webb, M.J., Keeney, G.L., Lesnick, T.G. and Podratz, K.C. (2002) Surgical stage I endometrial cancer: Predictors of distant failure and death. Gynecologic Oncology, 87, 274-280. doi:10.1006/gyno.2002.6836

[23] Abu-Rustum, N.R., Gomez, J.D., Alektiar, K.M., Soslow, R.A., Hensley, M.L., Leitao Jr., M.M., Gardner, G.J., Sonoda, Y., Chi, D.S. and Barakat R.R. (2009) The incidence of isolated paraaortic nodal metastasis in surgically staged endometrial cancer patients with negative pelvic lymph nodes. Gynecologic Oncology, 15, 236-238. doi:10.1016/j.ygyno.2009.07.016

[24] Fujimoto, T., Nanjyo, H., Nakamura, A., Yokoyama, Y., Takano, T., Shoji, T., Nakahara, K., Yamada, H., Mizunuma, H., Yaegashi, N., Sugiyama, T., Kurachi, H., Sato, A. and Tanaka, T. (2007) Para-aortic lymphadenectomy may improve disease-related survival in patients with multipositive pelvic lymph node stage IIIc endometrial cancer. Gynecologic Oncology, 107, 253-259. doi:10.1016/j.ygyno.2007.06.009

[25] Mariani, A., Webb, M.J., Galli, L. and Podratz, K.C. (2000) Potential therapeutic role of para-aortic lymphadenectomy in node positive endometrial cancer. Gynecologic Oncology, 76, 348-356. doi:10.1006/gyno.1999.5688

[26] Hoekstra, A.V., Kim, R.J., Small Jr., W., Rademaker, A.W., Helenowski, I.B., Singh, D.K., Schink, J.C. and Lurain, J.R. (2009) FIGO stage IIIC endometrial carcinoma: Prognostic factors and outcomes. Gynecologic Oncology, 114, 273-278. doi:10.1016/j.ygyno.2009.04.013

[27] Kilgore, L.C., Partridge, E.E., Alvarez, R.D., Austin, J.M., Shingleton, H.M., Noojin, F. and Conner, W. (1995) Adenocarcinoma of the endometrium: Survival comparisons of patients with and without pelvic node sampling. Gynecologic Oncology, 56, 29-33.

\section{doi:10.1006/gyno.1995.1005}

[28] Mohan, D.S., Samuels, M.A., Selim, M.A., Shalodi, A.D., Ellis, R.J., Samuels, J.R. and Yun, H.J. (1998). Long-term outcomes of therapeutic pelvic lymphadenectomy for stage I endometrial adenocarcinoma. Gynecologic Oncology, 70, 165-171. doi:10.1006/gyno.1998.5098

[29] Cragun, J.M., Havrilesky, L.J., Calingaert, B., Synan, I., Secord, A.A., Soper, J.T., Clarke-Pearson, D.L. and Berchuck, A. (2005) Retrospective analysis of selective lymphadenectomy in apparent early-stage endometrial cancer. Journal of Clinical Oncology, 23, 3668-3675. doi:10.1200/JCO.2005.04.144

[30] Trimble, E.L., Kosary, C. and Park, R.C. (1998) Lymph node sampling and survival in endometrial cancer. Gynecologic Oncology, 71, 340-343. doi:10.1006/gyno.1998.5254

[31] Chan, J.K., Cheung, M.K., Huh, W.K., Osann, K., Husain, A., Teng, N.N. and Kapp, D.S. (2006) Therapeutic role of lymph node resection in endometrioid corpus cancer: A study of 12,333 patients. Cancer, 107, 1823-1830. doi:10.1002/cncr.22185

[32] Chan, J.K., Wu, H., Cheung, M.K., Shin, J.Y., Osann, K. and Kapp, D.S. (2007) The outcomes of 27,063 women with unstaged endometrioid uterine cancer. Gynecologic Oncology, 106, 282-288. doi:10.1016/j.ygyno.2007.05.033

[33] Neubauer, N.L., Havrilesky, L.J., Calingaert, B., Bulusu, A., Bernardini, M.Q., Fleming, N.D., Bland, A.E. and Secord, A.A. (2009) The role of lymphadenectomy in the management of preoperative grade 1 endometrial carcinoma. Gynecologic Oncology, 112, 511-516. doi:10.1016/j.ygyno.2008.11.012

[34] Smith, D.C., Macdonald, O.K., Lee, C.M. and Gaffney D.K. (2008) Survival impact of lymph node dissection in endometrial adenocarcinoma: A surveillance, epidemiology, and end results analysis. International Journal of Gynecological Cancer, 18, 255-261. doi:10.1111/j.1525-1438.2007.01020.x

[35] Todo, Y., Kato, H., Kaneuchi, M., Watari, H., Takeda, M. and Sakuragi, N. (2010) Survival effect of para-aortic lymphadenectomy in endometrial cancer SEPAL study: A retrospective cohort analysis. Lancet, 375, 1165-1172. doi:10.1016/S0140-6736(09)62002-X

[36] Lowery, W.J. Gehrig, P.A., Ko, E., Secord, A.A., Chino, J. and Havrilesky, L.J. (2012) Surgical staging for endometrial cancer in the elderly-Is there a role for lymphadenectomy? Gynecologic Oncology, 126, 12-15. doi:10.1016/j.ygyno.2012.05.003

[37] Chan, J.K., Urban, R., Cheung, M.K., Shin, J.Y., Husain, A., Teng, N.N., Berek, J.S., Walker, J.L., Kapp, D.S. and Osann K. (2007) Lymphadenectomy in endometrioid uterine cancer staging: How many lymph nodes are enough? A study of 11,443 patients. Cancer, 109, $2454-$ 2460. doi:10.1002/cncr.22727

[38] Kitchener, H., Swart, A.M., Qian, Q., Amos, C. and Parmar, M.K. (2009) Efficacy of systematic pelvic lymphadenectomy in endometrial cancer (MRC ASTEC trial): A randomised study. Lancet, 373, 125-136. doi:10.1016/S0140-6736(08)61766-3 
[39] Walker, J.L., Piedmonte, M.R., Spirtos, N.M., Eisenkop, S.M., Schlaerth, J.B., Mannel, R.S., Spiegel, G., Barakat, R., Pearl, M.L. and Sharma, S.K. (2009) Laparoscopy compared with laparotomy for comprehensive surgical staging of uterine cancer: Gynecologic Oncology Group Study LAP2. Journal of Clinical Oncology, 27, 53315336. doi:10.1200/JCO.2009.22.3248

[40] Lutman, C.V., Havrilesky, L.J., Cragun, J.M., Secord, A.A., Calingaert, B., Berchuck, A., Clarke-Pearson, D.L. and Soper, J.T. (2006) Pelvic lymph node count is an important prognostic variable for FIGO stage I and II endometrial carcinoma with high-risk histology. Gynecologic Oncology, 102, 92-97. doi:10.1016/j.ygyno.2005.11.032

[41] Panici, P.B., Maggioni, A., Hacker, N., Landoni, F., Ackermann, S., Campagnutta, E., Tamussino, K., Winter, R., Pellegrino, A., Greggi, S., Angioli, R., Manci, N., Scambia, G., Dell’Anna, T., Fossati, R., Floriani, I., Rossi, R.S., Grassi, R., Favalli, G., Raspagliesi, F., Giannarelli, D., Martella, L. and Mangioni, C. (2005) Systematic aortic and pelvic lymphadenectomy versus resection of bulky nodes only in optimally debulked advanced ovarian cancer: A randomized clinical trial. Journal of the $\mathrm{Na}$ tional Cancer Institute, 97, 560-566. doi:10.1093/jnci/dji102

[42] ASTEC Study Group, Kitchener, H., Swart, A.M., Qian, Q. and Parmar, M. (2009) Efficacy of systematic pelvic lymphadenectomy in endometrial cancer (MRC ASTEC trial): A randomised study. Lancet, 373, 125-136. doi:10.1016/S0140-6736(08)61766-3

[43] Creasman, W.T., Mutch, D.E. and Herzog T.J. (2010) ASTEC lymphadenectomy and radiation therapy studies: Are conclusions valid? Gynecologic Oncology, 116, 293294. doi:10.1016/j.ygyno.2009.10.065

[44] Frumovitz, M., Slomovitz, B.M., Singh, D.K., Broaddus, R.R., Abrams, J., Sun, C.C., Bevers, M. and Bodurka, D.C. (2004) Frozen section analyses as predictors of lymphatic spread in patients with earlystage uterine cancer. Journal of the American College of Surgeons, 199, 388-393. doi:10.1016/j.jamcollsurg.2004.05.258

[45] Quinlivan, J.A., Petersen, R.W. and Nicklin, J.L. (2001) Accuracy of frozen section for the operative management of endometrial cancer. BJOG, 108, 798-803.

[46] Shim, J.U., Rose, P.G., Reale, F.R., Soto, H., Tak, W.K. and Hunter, R.E. (1992) Accuracy of frozen-section diagnosis at surgery in clinical stage I and II endometrial carcinoma. American Journal of Obstetrics \& Gynecology, 166, 1335-1338.

[47] Pristauz, G., Bader, A.A., Regitnig, P., Haas, J., Winter, R. and Tamussino, K. (2009) How accurate is frozen section histology of pelvic lymph nodes in patients with endometrial cancer? Gynecologic Oncology, 115, 12-17. doi:10.1016/j.ygyno.2009.07.007

[48] Khoury-Collado, F., Glaser, G.E., Zivanovic, O., Sonoda, Y., Levine, D.A., Chi, D.S., Gemignani, M.L., Barakat, R.R. and Abu-Rustum, N.R. (2009) Improving sentinel lymph node detection rates in endometrial cancer: How many cases are needed? Gynecologic Oncology, 115, 453455. doi:10.1016/j.ygyno.2009.08.026

[49] Mais, V., Peiretti, M., Gargiulo, T., Parodo, G., Cirronis,
M.G. amd Melis, G.B. (2010) Intraoperative sentinel lymph node detectionby vital dye through laparoscopy or laparotomy in early endometrial cancer. Journal of Surgical Oncology, 101, 408-412.

[50] Querleu, D., Leblanc, E. and Castelain, B. (1991) Laparoscopic pelvic lymphadenectomy in the staging of early carcinoma of the cervix. American Journal of Obstetrics \& Gynecology, 164, 579-581.

[51] Boruta II, D.M., Growdon, W.B., McCann, C.K., Garrett, L.A., del Carmen, M.G., Goodman, A. and Schorge, J.O. (2011) Evolution of surgical management of early-stage endometrial cancer. American Journal of Obstetrics \& Gynecology, 205, 1-6. doi:10.1016/j.ajog.2011.06.081

[52] Mourits, M.J., Bijen, C.B., Arts, H.J., ter Brugge, H.G., van der Sijde, R., Paulsen, L., Wijma, J., Bongers, M.Y., Post, W.J., van der Zee, A.G. and de Bock, G.H. (2010) Safety of laparoscopy versus laparotomy in early-stage endometrial cancer: A randomised trial. Lancet Oncology, 11, 763-771. doi:10.1016/S1470-2045(10)70143-1

[53] Bijen, C.B., de Bock, G.H., Vermeulen, K.M., Arts, H.J., ter Brugge, H.G., van der Sijde, R., Kraayenbrink, A.A., Bongers, M.Y., van der Zee, A.G. and Mourits, M.J. (2011) Laparscopic hysterectomy is preferred over laparotomy in early endometrial cancer patients, however not cost effective in the very obese. European Journal of Cancer, 47, 2158-2165. doi:10.1016/j.ejca.2011.04.035

[54] Barnett, J.C., Havrilesky, L.J., Bondurant, A.E., Fleming, N.D., Lee, P.S., Secord, A.A., Berchuck, A. and Valea, F.A. (2011) Adverse events associated with laparoscopy vs laparotomy in the treatment of endometrial cancer. American Journal of Obstetrics \& Gynecology, 205, 143. doi:10.1016/j.ajog.2011.03.012

[55] Walker, J.L., Piedmonte, M.R., Spirtos, N.M., Eisenkop, S.M., Schlaerth, J.B., Mannel, R.S., Barakat, R., Pearl, M.L. and Sharma, S.K. (2012) Recurrence and survival after random assignment to laparoscopy versus laparotomy for comprehensive surgical staging of uterine cancer: Gynecologic Oncology Group LAP2 Study. Journal of Clinical Oncology, 30, 695-700. doi:10.1200/JCO.2011.38.8645

[56] Ghezzi, F., Uccella, S., Cromi, A., Bogani, G., Robba, C., Serati, M. and Bolis, P. (2012) Lymphoceles, lymphorrhea, and lymphedema after laparoscopic and open endometrial cancer staging. Annals of Surgical Oncology, 19, 259-267. doi:10.1245/s10434-011-1854-5

[57] Diaz-Arrastia, C., Jurnalov, C., Gomez, G. and Townsend Jr., C. (2002) Laparoscopic hysterectomy using a computer-enhanced surgical robot. Surgical Endoscopy and Other Interventional Techniques, 16, 1271-1273.

[58] Paley, P.J., Veljovich, D.S., Shah, C.A., Everett, E.N., Bondurant, A.E., Drescher, C.W. and Peters III, W.A. (2011) Surgical outcomes in gynecologic oncolgy in the era of robotics: analysis of first 1000 cases. American Journal of Obstetrics \& Gynecology, 204, 551. doi:10.1016/j.ajog.2011.01.059

[59] Goel, M., Zollinger, T.W. and Moore, D.H. (2011) Surgical staging of endometrial cancer: Robotic versus open technique outcomes in a contemporary single surgeon series. Journal of Robotic Surgery, 5, 109-114. 
doi:10.1007/s11701-010-0239-2

[60] Boggess, J.F., Gehrig, P.A., Cantrell, L., Shafer, A., Ridgway, M., Skinner, E.N. and Fowler, W.C. (2008) A comparative study of 3 surgicalmethods for hysterectomy with staging for endometrial cancer: Robotic assistance, laparoscopy, laparotomy. American Journal of Obstetrics \& Gynecology, 199, 360. doi:10.1016/j.ajog.2008.08.012
[61] Hoekstra, A.V., Jairam-Thodla, A., Rademaker, A., Singh, D.K., Buttin, B.M., Lurain, J.R., Schink, J.C. and Lowe, M.P. (2009) The impact of robotics on practice management of endometrial cancer: Transitioning from traditional surgery. International Journal of Medical Robotics and Computer Assisted Surgery, 5, 392-397. doi:10.1002/rcs.268 